\title{
EL PROCESO DE REVISIÓN EN CALDERÓN, VISTO A TRAVÉS DE SUS COMEDIAS AUTÓGRAFAS
}

\author{
Erik Coenen \\ Instituto del Teatro de Madrid \\ Universidad Complutense de Madrid \\ Facultad de Filología, Edificio D \\ Avenida Complutense s.n. \\ 28040 Madrid. España \\ ewcoenen@ucm.es
}

Bastantes de los autógrafos conservados de las comedias de Calderón han sido analizados, más o menos minuciosamente, en los estudios preliminares a sus respectivas ediciones críticas, pero hoy por hoy no existe un catálogo analítico del conjunto comparable con el admirable trabajo llevado a cabo por Marco Presotto en torno a los autógrafos de Lope, aunque el catálogo de Alviti de las comedias escritas en colaboración, que incluye cinco en las que participó Calderón, es un paso en la buena dirección ${ }^{1}$. Estas páginas, desde luego, no pueden aspirar a suplir esta carencia. Su propósito, más humilde, es llegar a entender mejor el conjunto por medio del examen de ciertas peculiaridades de manuscritos individuales. Muy en especial, en lo que se refiere a lo que pueden decirnos sobre el proceso de composición o, al menos, sobre el proceso de revisión de los textos.

¿Cómo se compone una comedia? Conocemos el razonable consejo que brinda Lope en el Arte nuevo de iniciar la labor escribiendo el argumento en prosa (v. 211), antes de proceder a la redacción verbal de la

${ }^{1}$ Presotto, 2000 y Alviti, 2006. Cruickshank (1970) estudió el conjunto para analizar la letra de Calderón y su evolución. Bastantes observaciones generales se encuentran en Ruano de la Haza, 2000. 
comedia propiamente dicha; pero muy poco sabemos del proceso subsiguiente. Sería precipitado suponer que los poetas empezaran redactando el principio de la escena inicial y acabaran componiendo el último verso del desenlace. De hecho, no sería del todo descabellado suponer que procedieran a la inversa, empezando por la escena final y luego retrocediendo hasta llegar a la primera. O podrían haber escrito primero los discursos principales y luego haberlos ido uniendo con los diálogos, o haber variado su método según el caso. No lo sabemos, pero lo que sí creo que podemos, al menos, intuir, es que a nivel de las estrofas, el proceso de composición a menudo no es, por así decirlo, lineal, desde el primer verso al último. Una ley no escrita de la poesía rimada estipula que, para evitar la sensación de ripio, conviene que la palabra-rima más rebuscada sea la inicial y la más «natural» la final; y esa palabra, la que con más naturalidad procede del tema o de las circunstancias, será generalmente la primera en surgir en la mente del poeta. Basta ver unos cuantos ejemplos entresacados casi arbitrariamente de la obra de Calderón para ver cómo la composición de una estrofa tiene muy a menudo su inicio en el final de la misma. Pienso, por ejemplo, en esta estrofa, con la que Celia, en La selva confusa, anuncia ante la perplejidad del Duque de Mantua la verdadera identidad del pescador que vive en su casa:

\author{
y porque se certifique \\ la verdad de un desengaño \\ sin locura y sin engaño: \\ el pescador es Fadrique. (vv. 2929-2932)
}

Sin duda, fue el nombre del protagonista, Fadrique, y probablemente el octosílabo final ya compuesto en su totalidad, lo que le sugirió a Calderón la palabra-rima "certifique» (lo cual no es rémora para que haya probado también otras soluciones, como "publique»), y no al revés; la redondilla, pues, se compuso con toda probabilidad partiendo de su verso final y tal vez después rellenando entre el verso primero y último. Pienso, asimismo, en una décima de En la vida todo es verdad y todo mentira, que elabora el tópico de la mujer como "pequeño cielo», y que termina así:

y siendo el hombre en el suelo breve mundo, en su azul velo bien que vi a la mujer fundo; pues si el hombre es breve mundo, la mujer es breve cielo. (vv. 864-868) 
Parece claro que fue la antítesis final lo que hizo a Calderón buscar rimas en -undo y -elo, es decir, lo que le hizo iniciar la composición de la décima, o al menos la segunda mitad de esta, por el final.Y parece claro que la elección de la metáfora "azul velo» es posterior a la elección de la palabra final «cielo». No pretendo reducir el proceso de composición a un esquema excesivamente simple y ordenado, pues sin duda se produce en la mente del poeta y sobre el papel un proceso de gradual acomodación mutua entre las partes de la estrofa, y entre las partes y el todo; sólo quiero señalar que este proceso no dejó apenas rastro en los manuscritos autógrafos que conservamos, hecho que debe asombrarnos si creemos que estos manuscritos son primeros borradores.

Una ausencia semejante de huellas del proceso de composición resulta más llamativa aún en el caso, ya no de redondillas o décimas, sino de un soneto, pues un artefacto verbal de tal complejidad estructural nadie la sabe producir "de repente» si no es aquel gracioso lopesco de La niña de plata. En el mundo real, los sonetos no se improvisan sino que se construyen; pero el soneto de Calderón que figura en el folio 45r del autógrafo de La selva confusa no ha dejado indicio alguno de su gradual construcción desde la nada, si no es un pequeño borrón insustancial en el inicio del segundo verso, que parece más que nada la corrección de un desliz al copiar el texto (probablemente Calderón empezó escribiendo «vella Jacina», y después de escribir "ve» ya se dio cuenta de que el texto decía «Jacinta» a secas). Si el manuscrito fuera un primer borrador, habría que concluir que Calderón era capaz de componer sus sonetos de un tirón, empezando por el verso primero y acabando por el último, sin vacilar ni modificar nada sobre la marcha.

Otros ejemplos elocuentes se hallan en el magnífico monólogo de Focas al principio de En la vida todo es verdad y toda mentira. Sus versos 41-74 constituyen, al menos con la puntuación de la edición de Cruickshank, una única frase de 34 versos de sintaxis alambicada, pero no hay en el autógrafo nada que revele la labor llevada a cabo por el poeta para componerlos; sólo dos pequeñísimas enmiendas, realizadas casi seguramente en un momento posterior a la redacción del manuscrito. Ejemplos similares son fáciles de entresacar de los otros autógrafos que conservamos de Calderón.

Podría pensarse que Calderón fuera un genio de la composición mental; que compusiera en su mente la estrofa o la tirada de romance, o incluso el soneto entero, antes de pasarlo al papel. A fin de cuentas, 
escribir era laborioso, el papel era costoso, la memoria se entrenaba más que hoy y la oralidad era una parte más integrada de la cultura literaria. Pero cuesta creer en tal explicación. Los manuscritos suelen contener errores que parecen ser de transcripción, no de redacción. Ejemplos son «mis aplausos» por «tus aplausos» (El gran príncipe de Fez, 7v); «açechado» por «açechando» (En la vida todo es verdad y todo mentira, fol. $49 \mathrm{v}$, sin enmendar); «muera» por "muerta» (Yerros de Naturaleza y aciertos de la Fortuna, fol. 29v, sin enmendar), "y engendrar su muerte engendra» por "y esperar su muerte engendra» (La selva confusa, fol. $5 \mathrm{r}$, sin enmendar), etcétera. En ninguno de ellos he encontrado lo que podríamos llamar elementos auxiliares al proceso de composición, elementos como, por ejemplo, lo que sí encontramos en aquel borrador que se conserva del romance que Lorca dedicó a Córdoba: una lista de 17 palabras con la asonancia $u$ - $a$, apuntadas para su eventual empleo en el poema ${ }^{2}$. Más importante para lo que pretendo demostrar aquí es el hecho de que en las revisiones marginales, que, por su ubicación sobre la página y muchas veces también por el color de la tinta, pertenecen claramente a una fase posterior a la redacción del manuscrito, sí se encuentran indicios de vacilaciones, de dudas, de sucesivos cambios de parecer por parte del poeta; indicios que en el cuerpo del texto brillan precisamente por su ausencia. Este contraste entre la seguridad que muestra el texto de los manuscritos en su redacción primera, y la vacilación que evidencian las revisiones autorales posteriores, es lo que con más fuerza demuestra, a mi modo de ver, que los manuscritos no pueden ser sino transcripciones de borradores anteriores, luego sometidos a un proceso más o menos extenso de revisión.

Sirva de ejemplo el primer folio del autógrafo de Calderón de la tercera jornada de Polifemo y Circe $^{3}$. En su recto tiene 18 versos, de los cuales tres aparecen enmendados. Así, en el segundo verso, que rezaba «ave de la espuma leue», han sido tachados «ave» y «la», y por encima de la primera tachadura el poeta escribió «pájaro», convirtiendo el verso en "pájaro de espuma leve». En el verso onceno, que rezaba "ombres armados aborta», tachó «armados» y escribió encima «a la tierra». Sobre estas dos modificaciones del texto hay que recalcar, no sólo el que, en efecto, deben de ser el resultado de una revisión posterior del texto por parte del poeta, sino

${ }^{2}$ El autógrafo en facsímil, con transcripción de Rafael Martínez Nadal, en García Lorca , 1975, p. 191.

${ }^{3}$ Para este autógrafo, ver Alviti, 2006, pp. 111-118. 
también el que no son estrictamente necesarias: pretenden mejorar la calidad del texto, no corregir errores o incongruencias. Lo mismo se puede decir del tercer caso, que es el que confirma lo dicho en el párrafo anterior. Se produce en el cuarto verso, que inicialmente rezaba «monstruo de las dos especies». Calderón tachó «las» y escribió «sus» encima («monstruo de sus dos especies»); tachó «sus» y escribió «y» al principio del verso («y monstruo de dos especies»); y finalmente, cambiando de parecer de nuevo, tachó "y» y escribió otra vez «sus» debajo de las tachadas «las» y «sus», volviendo así a su primera enmienda. Es decir que llegó a enmendar, de manera relativamente intranscendente, tres veces sucesivas el mismo verso. Como anuncié antes, esto que he llamado «vacilación» del poeta, se produce, pues, en la fase de revisión del texto, y contrasta radicalmente con la total ausencia de vacilación en los dieciocho versos originales del folio. Sugiere en efecto que, mientras el texto anterior a las modificaciones fue copiado de un texto ya redactado, modificaciones pequeñas del texto como esta fueron más bien improvisadas al vuelo, a veces demasiado precipitadamente, provocando sucesivas modificaciones de lo ya modificado. De modo similar, en el autógrafo de En la vida todo es verdad y todo mentira, en ese mismo monólogo de Focas en el que Calderón poeta fue capaz de escribir una frase de 34 versos sin revisar ni multiplicar versiones, el poeta sí dejó huellas de múltiples soluciones y sucesivas tachaduras en el proceso posterior, mucho menos complejo, de retocar el texto (por ejemplo vv. $135-138,171$ y $322-325)^{4}$.

Es de notar que vacilaciones dentro de una revisión autoral como las señaladas son más frecuentes en los retoques de limitado alcance que en las revisiones más largas, donde más esperables serían. Sirvan de ejemplo los añadidos marginales relativamente extensos que insertó el poeta en Troya abrasada ${ }^{5}$, fol. 46v; La selva confusa, fol. $7 \mathrm{r}$; En la vida todo es verdad y todo mentira, fol. 41v; o La más hidalga hermosura ${ }^{6}$, fol. 54r. En estos casos, cabe suponer que el poeta redactó primero los versos añadidos en un folio aparte antes de trasladarlos al manuscrito que conservamos, en un afán de evitar crear un texto ilegible por el exceso de sucesivos cambios introducidos.

\footnotetext{
${ }^{4}$ Véanse las notas textuales de la edición de Cruickshank, pp. 169-170, o el propio autógrafo, fols. 3v, 4r y $6 \mathrm{r}$.

${ }^{5}$ Para este manuscrito, ver Northup, 1913, especialmente pp. 195-205, y Alviti, 2006, pp. 128-137.

${ }^{6}$ Esta comedia sigue, que yo sepa, sin contar con edición moderna. El manuscrito es descrito por Alviti, 2006, pp. 72-83.
} 
$\mathrm{Si}$, como he querido demostrar en lo anterior, los autógrafos que se conservan de las comedias de Calderón no reflejan el proceso de composición en su fase inicial, sería conveniente suavizar o relativizar la habitual dicotomía entre «borradores» o rough drafts y "copias en limpio» o fair copies. Los autógrafos de Calderón que he ido citando en estas páginas pueden ser considerados todos borradores por el hecho de contener añadidos, supresiones y enmiendas; pero también han de ser entendidos, al menos en un sentido importante, como copias en limpio. No son primeros borradores, manuscritos en los que se compuso el texto — con seguridad mucho más sucios y fragmentarios, acaso bastante caóticos y quién sabe si ininteligibles para cualquier persona que no fuera el propio poeta-, sino el producto final de un proceso de composición. Todos pretenden ofrecer un texto "definitivo» en la medida en que un texto dramático puede serlo, es decir, un texto acabado y legible para otra persona y adecuado para su fin, que fue, generalmente, su entrega a un autor de comedias. Insisto en esto porque en la bibliografia dedicada a manuscritos individuales se encuentran a menudo afirmaciones y expresiones que apuntan a lo que me parece un malentendido al respecto. Sobre el acto segundo manuscrito de Yerros de Naturaleza y aciertos de la Fortuna, por ejemplo, se ha dicho que «debe de ser un primer borrador, porque está literalmente cuajado de tachaduras y enmiendas» ${ }^{7}$; y sobre el autógrafo de El mágico prodigioso, que «it is evidently a first draft rather than a finished manuscript of the kind which would be delivered to a company of actors, for it contains a considerable number of erasures and corrections ${ }^{8}$. A mi entender, por más tachaduras y enmiendas que contengan, hay que considerarlos manuscritos finales, listos para ser entregados a una compañía.

Cierto, el autógrafo de El mágico prodigioso sirvió de base para una nueva versión, también "final», posterior, y lo mismo cabe decirse del manuscrito de El agua mansa ${ }^{9}$; y se conservan también manuscritos que, a la inversa, son reescrituras posteriores de una versión «final» anterior, como los parcialmente autógrafos de El mayor monstruo y Basta callar ${ }^{10}$. En todos estos casos, se trata de versiones diferentes para circunstancias

${ }^{7}$ Cotarelo, 1919, p. 48.

${ }^{8}$ Wardropper, 1982, p. 22.

${ }^{9}$ Ver la edición de las dos versiones de Arellano y García Ruiz, 1989.

${ }^{10}$ Para el caso de Basta Callar, ver Altamiranda, 1995, pp. 6-9, y Greer, 2001, pp. $12-14$. 
diferentes de representación. Calderón puede a veces haber tenido asimismo otros motivos para sacar copias en limpio, por ejemplo como obsequio a un noble protector, o como texto base para la imprenta ${ }^{11}$. Pero en circunstancias normales, no tenía por qué malgastar su tiempo en transcribir de nuevo de principio a fin, sin cambiar nada, un texto ya "acabado", pues para tales copias en limpio, o para los papeles de los actores, había copistas, apuntadores u otras personas más indicadas.

El que un manuscrito redactado por el propio poeta como mero soporte del texto contenga enmiendas de la mano del mismo poeta no debe sorprender a nadie. El poeta seguía siendo dueño de sus versos y nada le impedía volver a modificarlos: nada salvo el temor a acabar generando un texto tan caótico que otra persona no lo pudiera ya descifrar sin correr el peligro de equivocarse. Como ya señalé, resulta manifiesto que se trata, casi siempre, de intervenciones posteriores en un pasaje ya compuesto, realizadas por un escritor siempre atento a oportunidades para mejorar su texto. Sustitución de una palabra adecuada por otra más o menos equivalente que, al parecer, le satisfacía más; retoque lexical para reforzar una figura retórica; inserción de unos versos para aclarar algún aspecto o para proporcionar información que faltaba: es este el tipo de intervenciones que encontramos habitualmente, pero siempre sobre un texto ya escrito, ya cohesionado. Bien mirado, es natural que un texto creado fragmentariamente - como no puede ser de otra manera tratándose de un texto extenso en verso- exija, una vez transcrito parcial o íntegramente, una nueva lectura de seguido, para su revisión y enmienda, y es esto lo que parecen evidenciar los manuscritos de los que disponemos y los cambios que contienen.

Aparte de las enmiendas hay que mencionar, desde luego, los atajos, que en la gran mayoría de los casos parecen tener como único fin reducir la extensión del texto con vistas a los límites temporales de la representación. Como observa Cruickshank, los 14 segmentos de cierta extensión atajados en En la vida todo es verdad y todo mentira, comparten «their lack of effect upon the action [...] They are precisely the sort of passages that offer themselves for deletion if the play has to be cut» ${ }^{12}$.

${ }^{11}$ La pulcritud de la colección de 23 autógrafos de autos sacramentales en formato folio conservados en la Biblioteca Histórica Municipal de Madrid sugiere que este podría ser su caso, como propone Ruano de la Haza (1995, p. 59 y 2000, p. 5).

12 Cruickshank, 1971, p. Xv. 
Greer sugiere algo similar sobre los pasajes atajados en Basta callar ${ }^{13}$. En el caso de La selva confusa, los más de 3600 versos que había redactado el poeta eran claramente excesivos, y son muchos los pasajes recuadrados para su omisión; pero sorprendentemente, muchos de ellos, lejos de ser omitidos en la transmisión posterior, fueron conservados en una u otra de las sueltas que derivan del manuscrito o, en algunos casos, en ambas. Al parecer, un atajo que no fuera acompañado de la instrucción expresa «no» todavía podía acabar siendo incluido en la copia siguiente; por no hablar de los atajos que evidencian un cambio de parecer mediante un «sí» o un «dícense» al margen ${ }^{14}$. En el caso de la segunda jornada de $\mathrm{La}$ desdicha de la voz, mucho más larga que las otras dos, se atajaron tiradas de versos en muchos lugares, y los atajos van acompañados al margen con un número, al parecer en la misma tinta y en la letra de Calderón, que coincide con la cantidad de versos tachados ${ }^{15}: 4$ aquí, 6 allí, 12 en otro lugar, etcétera, hasta un total de 114 versos, lo cual demuestra a las claras que la finalidad de la supresión fue, efectivamente, reducir en extensión el texto. Como las "víctimas» de tales atajos, siendo aquellos segmentos que no inciden en la acción, suelen ser amplificaciones retóricas o líricas, o intervenciones de los graciosos, no pocas veces se sacrifican precisamente pasajes de mayor belleza lírica o humorismo. Cabe debatir hasta qué punto debe respetarse, en una edición moderna del texto, la «voluntad del autor» de suprimir estos versos, habiendo sido estos atajados sin otro criterio que el de acortar la duración de la función, acaso a instancias ajenas además.

Más que la «suciedad» de algunos de los autógrafos conservados, acaso debe sorprendernos la pulcritud de otros. Los de El agua mansa ${ }^{16}$ y El mejor amigo, el muerto ${ }^{17}$ (tercera jornada), por ejemplo, carecen en la gran mayoría de sus hojas de enmienda autoral alguna, o sólo deslices corregidos al copiar el texto (con una llamativa tendencia al gradual

${ }^{13}$ Greer, 2001, p. 14.

${ }^{14}$ Coenen, 2011, pp. 27-30.

${ }^{15}$ Sorprendentemente, se omite este dato y estos números en la por lo demás minuciosa edición paleográfica de Ebersole. Mason, en cambio, los señala en la introducción a la suya y postula asimismo que los atajos se hicieron «in the interests of brevity» (p. 9).

${ }^{16}$ Manuscrito estudiado por Orduna, 1982; Cruickshank, 1989; Arellano y García Ruiz, 1989; y Altamiranda, 1992.

${ }^{17}$ Manuscrito estudiado por Alviti, 2006, pp. 84-98. 
incremento de enmiendas conforme avanza el texto, por cierto ${ }^{18}$. Ello no quiere decir que Calderón en estos casos adoptara una actitud de «mero» copista, sino, plausiblemente, que se esforzara especialmente en evitar que sus enmiendas llegaran al texto copiado, o que trabajara sobre un primer borrador más elaborado que en otros casos.Y es que, sin lugar a dudas, Calderón pulía sus textos no sólo después de copiados, sino también en el mismo momento de «trasladarlos». Esta afirmación puede parecer imposible de sustentar sin recuperar los borradores previos que se han perdido; pero ciertas peculiaridades de algunos autógrafos nos permiten entrever que es así.

Pienso sobre todo en los diversos casos que conocemos, en los autógrafos tanto de comedias como de autos, de hojas pegadas encima de otras hojas. Examinaré dos de ellos. El primero es el folio 22v de En la vida todo es verdad y todo mentira que Cruickshank encargó despegar de una página que resultó contener la versión anterior del mismo texto ${ }^{19}$. El motivo por el que Calderón acudió al pegamento en este caso creo que es bastante claro: las revisiones que añadió habían acabado creando un texto tan caótico que un copista u otro usuario podría equivocarse fácilmente. Pues bien, en la copia «en limpio» del folio, no sólo se recogen las revisiones marginales de la primera, sino que se introducen nuevos cambios, que son los que interesan aquí. Este pasaje, por ejemplo:

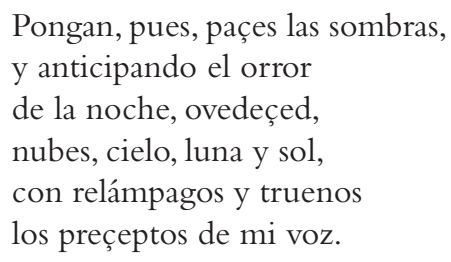

Pasó a leerse así (pongo en cursiva los cambios que no afectan al orden de los versos):

Pongan, pues, paçes las sombras, $\mathrm{y}$ anticipando el orror de la noche al pareçer,

${ }^{18}$ Ambos autógrafos incluyen algunos folios en mano ajena, que a lo mejor sustituyen precisamente los más enmendados del poeta, pero también cabe barajar otras explicaciones, como, por ejemplo, la eliminación de pasajes censurados.

${ }^{19}$ Cruickshank, 1971, p. XVIII. 
ovedezcan a mi voz, con relámpagos y truenos, nubes, cielo, luna y sol.

El final del folio, y de la jornada, reviste también modificaciones del texto. Pasemos por alto la conversión del nombre de Zoquete en Luquete, cambio que se hizo sistemáticamente en todo el manuscrito, para constatar aquí solo la introducción, en el texto de uno de los graciosos, de una deformación fonética que hace juego con el texto del otro:

Zoquete ¡Qué andalubio!

Sabañón ¡Qué turbión!

Se cambia a:

Zoquete ¡Qué andalubio!

SABAÑón ¡Qué antunbión!

Calderón probablemente acuñó la palabra «andaluvio» inicialmente como deformación cómica de «diluvio» habiendo ya elegido la palabra «turbión» por la asonancia; ahora caía en que la sinalefa le permitiría hacer al otro gracioso emplear el mismo prefijo, reforzando las cualidades retóricas del texto.

El desconcierto que causa el terremoto está bien reflejado en los fragmentarios textos que siguen a los citados en la versión temprana:

Focas ¡Libia!

Libia ¡Focas!

Focas ¡Cintia!

Cintia ¡Ysmenia!

SABAÑón ¡Ah, Zoquete!

ZOQUeTe ¡Sabañón!

Heraclio ¡Astolfo!

Astolfo ¡Heraclio!

Heraclio ¡Leonido! 
LOS TRES ¡Al monte!

Otros

¡A la población!

LIBIA

¡A la choça!

FOCAS

¡Al risco!

DAMA

¡Al llano!

Los tres versos iniciales, en los que nueve personajes son llamados por su nombre, expresan admirablemente la confusión y angustia del momento dramático, y resulta, a primera vista, extraño que Calderón suprimiera las últimas cinco de estas nueve intervenciones, dejando estos versos así:

$\begin{array}{lc}\text { Focas } & \text { ¡Libia! } \\ \text { Libia } & \text { ¡Focas! } \\ \text { Focas } & \text { ¡Cintia! } \\ \text { Cintia } & \text { ¡Ysmenia! } \\ \text { UnOS } & \text { ¡A la población! } \\ \text { Otros } & \text { ¡A la choça! } \\ \text { Otros } & \text { ¡Al risco! } \\ \text { Otros } & \text { ¡Al llano! }\end{array}$

Me temo que el motivo de la supresión fuera trivial: si no un mero desliz, el temor a no tener espacio suficiente para el texto entero, pues todavía tenían que caber los versos de Lisipo que concluyen el acto. Estos, por cierto, también modificados en relación con la versión copiada:

Lisipo Pues al tupido pavor embaraçadas las yras buscan todos su mansión, en lo que para dirá, otra vez que salga, el sol.

Lisipo Pues en tanta confusión embaraçadas las yras 
buscan todos su mansión,

en lo que paran dirá,

otra vez que salga, el sol.

También en el autógrafo de La desdicha de la voz hay una revisión textual llevada a cabo en un hoja nueva, pegado en la original (fol. $43 \mathrm{v})^{20}$. Se trata del final de la segunda jornada, que en el folio original queda inconcluso y que en el nuevo llega hasta el final de la acción, por lo que cabe sospechar que la nueva versión se hizo como parte de la tarea de acortar la jornada. Partes del texto se mantienen, pero, también en este caso, con modificaciones, como donde el folio original dice:

D. Pedro Pues yo me quiero perder, porque no e de estar oyendo que esté una yngrata cantando estándome yo muriendo.

D. JUAN ¡A,Veatriz, en qué peligro oy la desdicha te a puesto de tu voz!

Otavio

Salir no avéis.

Cierra aquesas puertas, Celio.

En el folio pegado se lee así, con un conjunto de cambios interrelacionados hacia el final del pasaje:

D. Pedro Pues yo me quiero perder, porque no e de estar oyendo que esté una yngrata cantando estándome yo muriendo.

Otavio No le dejéis.

D. JUAN iAy, Veatriz, en qué peligro te a puesto la desdicha de tu voz!

Otavio Cierra aquesas puertas, Celio.

Nada sustancial. Un aparte de Otavio a don Juan intercalado, y una reformulación que permite aludir de modo más literal al título de la co-

${ }^{20}$ Mason reproduce los diversos estadios del texto en su edición, 2003, pp. 219-250. 
media. Lo que importa es que el caso coincide con el de los versos de la duplicada página final de la primera jornada de En la vida todo es verdad y todo mentira en evidenciar que Calderón, al copiar sus propios versos, aprovechaba la oportunidad para retocarlos, aunque, eso sí, haciendo lo posible para evitar crear confusión en el manuscrito, y sin duda componiendo en un folio aparte siempre cuando la revisión era mínimamente compleja.

También en algunas hojas del autógrafo de El mágico prodigioso - no por casualidad, las que abarcan la disputa teológica entre Cipriano y el Demonio: auténtico reto imaginativo e intelectual para el poetaCalderón reescribió y volvió a retocar varios trozos, creando tal desorden que se vio impulsado a pasarlas a limpio de nuevo. En este caso, no pegó hojas encima de los folios inservibles, sino que insertó en el manuscrito dos hojas con el texto copiado, que son los fols. 7 y 8 , según la foliación antigua (¿de Calderón?), y 9 y 10, según la foliación moderna (la diferencia en la foliación estriba precisamente en que los folios inservibles no llegaron a ser numerados inicialmente) $)^{21}$. Los primeros seis versos del folio siguiente - fol. 9r (antigua) o 11r (moderna) - están tachados, por ser los mismos que concluyen la segunda hoja insertada: una prueba, me parece, de que las dos hojas fueron copiadas e insertadas en una fase posterior a la composición del manuscrito. Pues bien, también en este caso, Calderón, al pasar a limpio el texto revisado, volvió a modificarlo en algunos lugares. Omitió incluso tiradas enteras de versos, como esta (sigo la foliación moderna):

Cipriano Que sea distinto en personas
concedo, pero que aya
una cosa en que convengan
aquesas personas varias
y otra en que se encuentran, que es
la menor, e de negarla. (fol. 8 r)

Llevó a cabo también numerosos cambios menores, por ejemplo, al copiar «la fábrica gallarda / del mundo» como «la fábrica gallarda del hombre», o al modificar la despedida del Demonio y el aparte que la sigue. Así figura en la primera redacción:

${ }^{21}$ Lo señalan ya Morel-Fatio, 1877, quien incluye la redacción primitiva en su edición, pp. 248-250, y más recientemente, McKendrick, 1992, p. 6. 
Demonio El cielo os guarde.

([Ap.] Pues proposiciones falsas

por sutiles no convencen

tu yngenio, yo daré traza

de que dejes tus estudios

divertido en una rara

veldad, sacando ambicioso

de un efeto dos venganzas

entre Cipriano y Justina.

Infierno, ¡tocad al arma!)

En la «copia», se eliminan cuatro versos y se introducen varios cambios (la atribución del aparte final a Cipriano y no al Demonio es un error manifiesto). Nótese la insatisfacción del poeta con la forma de despedirse el Demonio:

Demonio Quedad en paz.

Cipriano ([Ap. $]$ Pues tanto tu estudio alcanza

yo haré que el estudio olvides,

suspendido en una rara

veldad, sacando ambicioso

de un efeto dos venganzas.)

Cabe añadir que en la princeps — en la Parte veinte de comedias nunca impresas de 1663-, figura de nuevo modificado el fragmento (respeto la puntuación del impreso):

Demonio Quedad en paz.

Pues tanto tu estudio alcanza:

Yo haré que el estudio oluides,

suspendido en una rara

veldad pues tengo licencia

de perseguir con mi rabia

a Iustina, sacaré

de un efecto dos venganzas. (fol. 5 r)

Una vez copiado el texto con tantas modificaciones, Calderón volvió a revisarlo, añadiendo, por ejemplo, al margen esta tirada de versos:

Luego si Dios todo es vista

qualquiera dios viera clara 
y distintamente un fin

$\mathrm{y}$, al verle, no asegurara

el que no avía de ser; luego

aunque sea deydad tanta,

distinta en personas debe

en la menor circunstancia

ser una sola en esencia

Demonio Ynportó para esa causa (fol. 9v)

No puedo examinar aquí en toda su complejidad la transformación del texto de fols. 7-8 en el de fols. 9-10 en este manuscrito, ni en los nuevos cambios introducidos —ipor el autor? - en el pasaje análogo de la princeps; baste este botón de muestra para atestiguar, de nuevo, el incansable afán del autor de mejorar sus textos.

Otro tipo de indicios de la incesante labor de revisión de Calderón nos los ofrece el manuscrito de Yerros de la Fortuna y aciertos de Naturaleza ${ }^{22}$, cuyo primer acto, así como parte del tercero, es de Antonio Coello acepto su autoría, aunque la letra en estas partes del manuscrito no es suya $^{23}$-; el resto es de Calderón. Se trata tal vez del manuscrito más «sucio» de Calderón ${ }^{24}$, con una frecuencia relativamente alta de enmiendas que parecen haberse producido ya en primera instancia, si bien son muchas más las que pertenecen a una fase posterior, de revisión. Algunas de las intervenciones autorales más interesantes para mi propósito se encuentran en el segmento final de la segunda jornada, escrito en romance en $e-o$. A ellas dedicaré las páginas finales de este trabajo.

La protagonista, Matilde, aparenta su propia muerte y luego suplanta a su hermano Polidoro en el poder, aprovechando su extraordinario parecido fisico - el «yerro de Naturaleza» del que habla el títuloDespués de haber asumido con éxito la identidad de su hermano ante los consejeros de este, pronuncia para sí unos versos que luego fueron atajados por el poeta; luego alguien — creo que el propio Calderónañadió un "sí» en cada margen, pero esos síes también acabaron siendo tachados — creo que otra vez por Calderón-. Sin lugar a dudas, estos

${ }^{22}$ Lo describen Northup, 1911, pp. 411-416 y Alviti, 2006, pp. 160-166; la edición de Juliá Martínez recoge en nota casi todos lo tachado y enmendado.

${ }^{23}$ Todos los catálogos y todos los estudiosos que se han ocupado de este manuscrito dan por sentado, erróneamente, que sí lo es.Ver Coenen, 2015, en prensa.

${ }^{24}$ Lo es al menos en comparación con los otros cuatro autógrafos de comedias colaboradas, como se deduce de los estadísticas al respecto que ofrece Alviti, 2006, passim. 
cambios de parecer guardan relación con el hecho de que los mismos versos fueron empleados unos 250 versos más abajo para concluir el acto. Por supuesto, Calderón no habrá querido que su personaje dijera dos veces los mismos versos y cabe suponer que estos le satisfacían como conclusión del acto. Ahora bien, el texto no es idéntico en los dos casos; reproduzco aquí las dos versiones, destacando las variantes en cursiva.

\author{
Ya soy Polidoro, ya \\ rey soy, ¡o rraros sucesos \\ de Naturaleza, o rraros \\ de la Fortuna portentos, \\ qué fáciles de creer \\ sois! Vien se ve en este ejemplo, \\ pues oy, quando por Matilde \\ Polonia hace sentimientos, \\ es el muerto Polidoro, \\ y cuando rinde trofeos \\ a Polidoro, es Matilde \\ la que sólo go[za] de ellos: \\ tan poca distancia ay \\ entre una viba y un muerto. \\ Mis esequias son. $\mathrm{j} \mathrm{O}$ rraros \\ prodigios, $o$ altos portentos, \\ qué fáciles de creer \\ sois! Vien se ve en este ejemplo, \\ pues hoy, quando por Matilde \\ Polonia hace sentimientos, \\ es el muerto Polidoro, \\ y oy, cuando rinde trofeos \\ a Polidoro, es Matilde \\ la que sólo goza de ellos: \\ tan poca distancia ay \\ entre una viba y un muerto. (fol. 41v)
}

Nótese que en la segunda versión, al no figurar la referencia anterior a «Naturaleza», Calderón elimina también la referencia a «la Fortuna» con la que hacía antítesis. Inserta un «hoy» que refuerza la organización retórica del texto, haciendo anáfora y paralelismo con los tres versos anteriores. Una vez más, pues, vemos cómo Calderón, al transcribir un texto propio, no lo hace mecánicamente, sino que aprovecha la opor- 
tunidad de revisarlo y pulirlo. Y el proceso siguió: justo arriba del penúltimo verso, insertó al margen los versos «tristeza me da mirar / que entre mis esequias reyno»; tachó luego el primer verso y escribió encima "pabor me ha dado pensar», en una propuesta manifiesta de mejora. Estas intervenciones, a su vez, le obligaron a modificar levemente los dos versos finales, que quedaron como «y que ay tan poca distancia entre una vida y un muerto», donde a su vez "vida» debe ser error por «viva». El resultado para los versos finales es, pues:

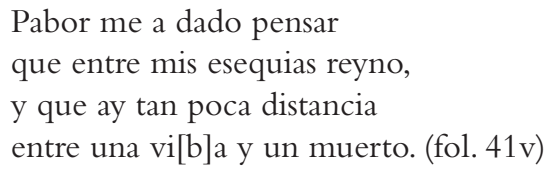

Pabor me a dado pensar que entre mis esequias reyno, y que ay tan poca distancia entre una vi[b]a y un muerto. (fol. 41v)

En otro lugar del mismo manuscrito, Calderón, al parecer, decidió retrasar la salida en escena del gracioso Tabaco, habiendo escrito ya al menos trece versos que acompañan esta salida. Parece casi seguro que escribió en realidad más que los trece versos tachados, pues estos llegan, sin acabar la frase, justo al final del folio $38 \mathrm{v}$, por lo que cabe suponer que lo tachado seguía en el folio siguiente, que fue desechado (habría que desencuadernar las hojas para confirmar esta conjetura); incluso no se puede descartar que Calderón reescribiera toda la parte final del segundo acto, reorganizando el desarrollo de la acción y deshaciéndose de los folios originales. Sea como fuere, como ocurrió en las hojas copiadas y pegadas de En la vida todo es verdad y todo mentira y La desdicha de la voz, como ocurrió en las hojas repetidas del autógrafo de El mágico prodigioso, y como sucedió con los versos de Matilde antes citados, la segunda vez que Calderón escribió los trece versos que aquí nos ocupan, los retocó. Comparemos los versos finales de las dos versiones, que es donde se produjeron los cambios (el verso que va entre corchetes lo reconstruyo por conjetura, pues no lo llegó a escribir Calderón en el manuscrito conservado, o lo escribió en el folio siguiente):

porque era una infanta hija

de diocleciano y denero; un sayón se merendaba [como si fuera un buñuelo] (fol.38v) 
porque era una infanta hija

del emperador enero;

sayones se merendaba

como si fueran buñuelos (fol. 40r)

Fuesen cuales fuesen los motivos de Calderón para suprimir la referencia a un emperador notorio por su persecución de los cristianos, y para poner sayón en plural, lo que parece bastante claro es que estamos ante otra prueba más de que Calderón, al copiar sus textos, los volvía a limar casi por sistema ${ }^{25}$.

Una vez definitivamente salido en escena Tabaco, comparte escena con Matilde, Fisberto y Federico. Calderón reutilizó en este segmento unos versos que ya figuran antes, pero tachados, así:

Federico ¿Qué burlas, señor...

Fisberto

¿Qué juegos...

Federico $\quad \ldots$ de la Fortuna son estas?

Fisberto $\quad \ldots$ de la pribança son estos? (fol. $39 \mathrm{v}$ )

De hecho, seis versos más arriba en el mismo fol. $39 \mathrm{v}$, el poeta ya había iniciado los mismos versos, llegando a escribir sólo «qué burlas, señor...", adjudicando estas palabras, por cierto, a Fisberto y no a Federico como en el segundo intento. Finalmente, cuando los citados versos encontraron su lugar final, estando ya Tabaco en escena, quedaron así:

FEDERICO ¿Qué enigmas, señor, qué cifras...

Fisberto ¿Qué burlas, señor...

ТАВACO

¿Qué juegos...

Federico $\quad$... de la Fortuna son estas?

FisBerto de la pribança son estos?

TABACO $\quad \ldots$ de las manos son estotras? (fol. 41r)

${ }^{25}$ A una conclusión similar llega Ruano de la Haza a raíz de una «rápida mirada» a las copias que sacó Calderón de algunos autos sacramentales suyos: "Calderón no se limitaba simplemente a transcribir su texto base, sino que seguía puliendo, alterando, añadiendo y omitiendo versos; es decir, produciendo versiones más o menos diferentes y divergentes de las que escribió originalmente» (2000, p. 7). 
Se aprecia el intento de incorporar a Tabaco en un diálogo en el que, en una versión anterior, no estaba presente. Obsérvense los errores de concordancia que generó la modificación, al parecer no notados por el autor y resultados de una intervención quizá descuidada, irreflexiva, precipitada: puede que el tiempo apremiara, y que ya de por sí Calderón no se preocupara mucho por las comedias escritas en colaboración ${ }^{26}$.

De modo que ejemplos sacados de cuatro autógrafos diferentes atestiguan que Calderón, al copiar sus textos, los limaba y modificaba casi por sistema, sin considerarlos nunca definitivos ni inmejorables; hecho que tal vez puede explicar al menos en parte, dicho sea de paso, la multiplicidad de versiones conservadas de algunas comedias suyas (El astrólogo fingido, La devoción de la cruz, Judas Macabeo, Para vencer amor querer vencerlo, La vida es sueño, etc.).

Me he detenido en esta cuestión porque los ejemplos que he dado figuran entre las pocas pruebas que nos permiten entrever lo que sucedía cuando Calderón transcribía sus versos desde sus borradores primitivos a los manuscritos que entregaba a las compañías. Sin lugar a dudas los modificaba en muchos lugares conforme los copiaba. Así se explican tal vez aquellas enmiendas, no muy frecuentes, que por su posición sobre la hoja parecen haber sido llevadas a cabo, no en una fase posterior, sino al vuelo. De modo que también estas, si no me equivoco, no reflejan el proceso inicial de composición sino el proceso de revisión, ejecutada, en este caso, en el mismo momento de copiar el texto, allí donde se produce la parte más fascinante y a la vez inaccesible del proceso: en la mente del poeta. Si «borrador» se define como «escrito de primera intención, en que se hacen o pueden hacerse adiciones, supresiones o enmiendas» (DRAE), habría que concluir que sólo la segunda parte de la definición es aplicable a los manuscritos autógrafos que se conservan de Calderón, pues ofrecen un texto "de segunda intención", ya retocado y tal vez - como sugiere el caso de Yerros de Naturaleza y aciertos de la Fortuna- reorganizado.

${ }^{26}$ Por otra parte, el manuscrito de Yerros de Naturaleza ofrece indicios de una radical reorganización precipitada del texto de la jornada tercera incluso después de su entrega a la compañía, que abordaré en otro lugar. 


\section{BiBLIOGRAFÍA}

Altamiranda, Daniel, «Descripción del manuscrito», en El agua mansa. Comedia autógrafa de Pedro Calderón de la Barca. Estudios dirigidos y editados de Lilia E. F. de Orduna, Kassel, Reichenberger, 1993, pp. 1-4.

Altamiranda, Daniel, «Estudio textual», en Calderón de la Barca, Pedro, Basta callar, ed. Daniel Altamiranda, Kassel, Reichenberger, 1995, pp. 54-79.

Alviti, Roberta, I manoscritti autografi delle commedie del "Siglo de Oro" scritte in collaborazione. Catalogo e studio, Alinea'Oro-Bibliografica, 48, Firenze, Alinea, 2006.

Arellano, Ignacio y Víctor García Ruiz, «El manuscrito autógrafo de El agua mansa», en Calderón de la Barca, Pedro, El agua mansa / Guárdate del agua mansa, ed. Ignacio Arellano y Víctor García Ruiz, Kassel, Reichenberger, 1989 , pp. 55-63.

Calderón de la Barca, Pedro, El agua mansa, manuscrito, Barcelona, Institut del Teatre.

Calderón de la BArca, Pedro, El agua mansa / Guárdate del agua mansa, ed. Ignacio Arellano y Víctor García Ruiz, Kassel, Reichenberger, 1989, pp. $55-63$.

Calderón de la Barca, Pedro, Basta callar, Ms. Res. 91, BNE.

Calderón de la Barca, Pedro, La desdicha de la voz, Ms. Res. 108, BNE.

Calderón de la Barca, Pedro, La desdicha de la voz, ed. Alva V. Ebersole, Madrid, Castalia, 1970.

Calderón de la Barca, Pedro, La desdicha de la voz, ed. Robert T. Mason, Liverpool, Liverpool University Press, 2003.

Calderón de la Barca, Pedro, En la vida todo es verdad y todo mentira, Ms. Res. 87, BNE.

Calderón de la Barca, Pedro, En la vida todo es verdad y todo mentira, ed. Don Cruickshank, London, Tamesis, 1970.

Calderón de la Barca, Pedro, El gran príncipe de Fez, Ms. Res. 100, BNE.

Calderón de la Barca, Pedro, La más hidalga hermosura (jornada tercera), en Juan de Zavaleta, Francisco de Rojas Zorrilla y Pedro Calderón de la Barca, La más hidalga hermosura, manuscrito, Barcelona, Institut del Teatre.

Calderón de la Barca, Pedro, El mágico prodigioso, Ms.Vitr 7 / 1, BNE.

Calderón de la Barca, Pedro, El mágico prodigioso, en Parte veinte de comedias varias nunca impressas, compuestas por los meiores ingenios de España, Madrid, Imprenta Real, 1663.

Calderón de la Barca, Pedro, El mágico prodigioso. A composite edition, ed. Melveena McKendrick, Oxford, Clarendon, 1992.

Calderón de la Barca, Pedro, El mágico prodigioso. Comedia famosa de Don Pedro Calderón de la Barca publiée d'après le manuscrit original de la bibliothèque du duc d'Osuna, ed. Alfred Morel-Fatio, Paris / Madrid, Heilbronn, 1877. 
Calderón de la Barca, Pedro, El mayor monstruo del mundo, Ms. Res. 79, BNE.

Calderón de la Barca, Pedro, Polifemo y Circe (jornada tercera), en Antonio Mira de Amescua (atr.), Juan Pérez de Montalván, y Pedro Calderón de la Barca, Polifemo y Circe, Ms. Res. 83, BNE.

Calderón de la Barca, Pedro, La selva confusa, Ms. Res. 75, BNE.

Calderón de la Barca, Pedro, La selva confusa, ed. Erik Coenen, Kassel, Reichenberger, 2011.

Calderón de la Barca, Pedro, Troya abrasada (jornadas segunda y tercera), en Juan de Zavaleta y Pedro Calderón de la Barca, Troya abrasada, Ms. Res. 78, BNE.

Calderón de la Barca, Pedro, Yerros de Naturaleza y aciertos de la Fortuna (jornada segunda y parte de la tercera), en Antonio Coello y Pedro Calderón de la Barca, Yerros de Naturaleza y aciertos de la Fortuna, Ms. 14778, BNE.

Coenen, Erik, "The Early Transmission of the Text», en La selva confusa, ed. Erik Coenen, Kassel, Reichenberger, 2011, pp. 12-48.

Coenen, Erik, "Yerros de Naturaleza y el copista que no fue Antonio Coello», 2015, en prensa.

Cotarelo y Mori, Emilio, Don Antonio Coello y Ochoa, Madrid, Tip. de la Revista de Archivos, Bibliotecas y Museos, 1919.

Cruickshank, Don W., "Calderón's handwriting», en Modern Language Review, LXV, 1970, pp. 65-77.

Cruickshank, Don W., «Introducción», en Pedro Calderón de la Barca, En la vida todo es verdad $y$ todo mentira, ed. Don W. Cruickshank, London, Tamesis, 1971, pp. XI-CXXXIII.

Cruickshank, Don W., «Notes on Calderonian Chronology and the Calderonian Canon", en Estudios sobre Calderón y el Teatro de la Edad de Oro. Homenaje a Kurt y Roswitha Reichenberger, Barcelona, PPU, 1989, pp. 19-36.

Ebersole, Alva V., "Comentario del manuscrito», en Pedro Calderón de la Barca, La desdicha de la voz, ed. Alva V. Ebersole, Madrid, Castalia, 1970, pp. XV-XVIII.

García Lorca, Federico, Autógrafos, vol. I, ed. Rafael Martínez Nadal, Oxford, Dolphin, 1975.

Greer, Margaret, «Textual history», en Pedro Calderón de la Barca, Basta Callar, ed. Margaret Greer, Ottawa, Dovehouse, 2000, pp. 11-17.

Mason, Robert T., «The Autograph Manuscript», en Pedro Calderón de la Barca, La desdicha de la voz, ed. Robert T. Mason, Liverpool, Liverpool University Press, 2003, pp. 1-14.

McKendrick, Melveena, "The Autograph Manuscript and its Revision", en Pedro Calderón de la Barca, El mágico prodigioso, ed. Melveena McKendrick, Oxford, Clarendon, 1992, pp. 6-16. 
Northup, George T., "Los Yerros de Naturaleza y Aciertos de la Fortuna by Don Antonio Coello and Don Pedro Calderón de la Barca», en Romanic Review, I, 1910, pp. 411-425.

Northup, George T., «Troya abrasada de Pedro Calderón de la Barca, y Juan de Zabaleta», en Revue Hispanique, XXIX, 1913, pp. 198-346 (ed. de la comedia en pp. 250-346).

Orduna, Lilia Ferrario de, «Un manuscrito de Calderón y sus editores», Incipit, II, 1982, pp. 107-116.

Presotto, Marco, Le commedie autografe di Lope de Vega. Catalogo e studio, Kassel, Reichenberger, 2000.

Ruano de la Haza, José María, «Introducción», en Pedro Calderón de la Barca, Andrómeda y Perseo, ed.José María Ruano de la Haza, Kassel, Reichenberger, 1995, pp. 11-134.

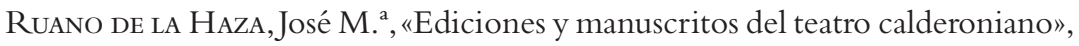
en Estado actual de los estudios calderonianos, ed. Luciano García Lorenzo, Kassel, Reichenberger, 2000, pp. 1-34.

WARDROPPER, Bruce, «The Manuscript and the Early Editions», en Calderón de la Barca, Pedro, The Prodigious Magician / El mágico prodigioso, Madrid, José Porrúa Turanzas, 1982, pp. 22-25. 